\title{
Terminalia ferdinandiana Exell. Kino Extracts have Anti-Giardial Activity and Inhibit Caco2 and HeLa Cancer Cell Proliferation
}

\author{
Joseph Shalom ${ }^{1,2}$, Paran Rayan ${ }^{1,2}$, Reece Courtney ${ }^{1,2}$, Pauline Ann McDonnell ${ }^{2}$, Ian Edwin Cock ${ }^{1,2^{*}}$ \\ 'Environmental Futures Research Institute, Nathan Campus, Griffith University, 170 Kessels Rd, Nathan, Queensland 4111, AUSTRALIA. \\ ${ }^{2}$ School of Natural Sciences, Nathan Campus, Griffith University, 170 Kessels Rd, Nathan, Queensland 4111, AUSTRALIA.
}

\begin{abstract}
Introduction: Terminalia ferdinandiana Exell. is an endemic Australian plant which is known for its exceptionally high antioxidant content. The fruit is a nutritional food and the leaves and kinos were used in Australian Aboriginal medicine to treat a variety of diseases. However, T. ferdinandiana kinos remain largely unexamined for their medicinal properties. Methods: Solvent extracts were prepared from $T$. ferdinandiana kinos and their antiproliferative activities against $G$. duodenalis and were determined by direct enumeration. The anti-proliferative activities against HeLa and Caco2 cancer cell lines were determined using an MTS based cell assay. Toxicity was determined using the Artemia franciscana nauplii bioassay. Results: Methanolic and aqueous T. ferdinandiana kino extracts were strong inhibitors of $\mathrm{CacO} 2$ and HeLa cell proliferation, with $\mathrm{IC}_{50}$ values substantially below $1000 \mu \mathrm{g} / \mathrm{mL}$. In contrast, the mid to lower polarity solvent extractions (ethyl acetate, chloroform and hexane) were less potent inhibitors of cell proliferation. The aqueous and methanolic extracts were also effective inhibitors of $G$. duodenalis proliferation, albeit with slightly higher $\mathrm{IC}_{50}$ values than against the Caco2 and HeLa cells. All T. ferdinandiana kino extracts were nontoxic in the Artemia franciscana bioassay, with $\mathrm{LC}_{50}$ values substantially
\end{abstract}

$>1000 \mu \mathrm{g} / \mathrm{mL}$. Conclusion: The antiproliferative activity of the T. ferdinandiana kino extracts against G. duodenalis and the HeLa and Caco2 cancer cell lines indicates their potential in the treatment and prevention of giardiasis and some cancers.

Key words: Kakadu plum, Combretaceae, Giardia duodenalis, Anticancer activity, Antiproliferative activity, Caco2, HeLa, Apoptosis.

\section{Correspondence:}

Dr. I.E. Cock,

'Environmental Futures Research Institute, Nathan Campus, Griffith University, 170 Kessels Rd, Nathan, Queensland 4111, AUSTRALIA.

${ }^{2}$ School of Natural Sciences, Nathan Campus, Griffith University, 170 Kessels Rd, Nathan, Queensland 4111, AUSTRALIA.

Tel: +61737357637

E-mail: I.Cock@griffith.edu.au

DOI: $10.5530 /$ pc. 2018.2 .13

\section{INTRODUCTION}

Plants of the genus Terminalia (family Combretaceae) are amongst the most widely used plants globally for traditional medicine purposes. ${ }^{1}$ Many species have antibacterial, antifungal, antiprotozoal, antiviral, antidiarrheal, analgesic, antimalarial, antioxidant, anti-inflammatory and anticancer activities. Some species also have wound healing and cardiovascular effects. Terminalia spp. are characterised by their high antioxidant contents, which may contribute to their therapeutic properties. Several individual species (and traditional medicine combinations containing Terminalia spp.) have been reported to have potent anticancer properties. The Ayurvedic medicine Triphala (which contains Terminalia belliricia (Gawrtn.) Roxb. And Terminalia chebula (Retz.) Lyons) has cytotoxic effects against thymic lymphoma cells, ${ }^{2,3}$ human breast cancer cell lines, ${ }^{2,4}$ human prostate cancer cell lines and human pancreatic cancer cell lines. ${ }^{5}$ Conversely, the cytotoxic effects of Triphala are negligible in normal cell lines. ${ }^{2,3}$ T. belliricia extracts have also demonstrated growth inhibitory effects towards human A549 lung cancer cell lines and HepG2 hepatocarcinoma cell lines without the presence of the other plant components present in Triphala. ${ }^{6}$ T. belliricia extracts also have synergistic effects with conventional anticancer chemotherapeutics, enhancing the cytotoxic activity of cisplatin and doxorubicin towards the cancer cell lines, indicating their potential as synergistic cancer chemotherapeutic agents.

The Australian species Terminalia ferdinandiana Exell. also has antiproliferative activity against a panel of cancer cell lines. ${ }^{7,8} T$. ferdinandiana fruit extracts activate caspase-7, caspase- 9 and poly (ADP-ribose) polymerase (PARP), indicating that apoptosis induction is via an intrinsic pathway. ${ }^{8}$ Extracts prepared from the leaves of two other Australian Terminalia spp. (T. carpentariae and T. grandiflora) were also recently reported to be good inhibitors of $\mathrm{Caco} 2$ and HeLa cell proliferation. ${ }^{9}$ That study identified 3 lanostane and 2 pentacyclic triterpenoids in the extracts and postulated that they may contribute to this activity.

What all of the studies testing $T$. ferdinandiana extracts for anticancer activity have in common is that they focus on extracts produced from the fruit. This is perhaps understandable given the extremely high antioxidant capacities of $T$. ferdinandiana fruit and the reported correlation between antioxidant activity and antiproliferative properties in cancer cell lines. ${ }^{10,11}$ However, there is no record of the fruit of this species being used medicinally. Instead, the first Australians recognised their nutritional value and used the fruit as a food. In contrast, the therapeutic properties of the leaves and kinos were well known to the first Australians. ${ }^{1,12}$ Despite this, there is a lack of studies testing the leaves or kinos for their therapeutic properties. Several recent papers have reported on the antibacterial ${ }^{13-15}$ and anticancer properties ${ }^{7}$ of the leaves of this species and have shown that these extracts often have more potent therapeutic properties than extracts prepared from the fruit. However, there is a lack of studies on the medicinal properties of $T$. ferdinandiana kino extracts. Furthermore, we were unable to find any studies examining the anti-Giardial properties of extracts of either the leaves or kinos, despite reports of potent activity for fruit extracts. ${ }^{16}$ Our study aimed to address these gaps in the literature by testing $T$. ferdinandiana kino extracts against $G$. duodenalis trophozoites, and $\mathrm{Caco} 2$ colorectal and HeLa human cervical carcinoma cell lines. 


\section{MATERIALS AND METHODS}

\section{Plant source and extraction}

The Terminalia ferdinandiana Exell. kinos used in this study were supplied and verified by David Boehme of Wild Harvest, Northern Territory (Australia) and were collected from a single tree near Humpty Doo, Australia. Voucher specimens are stored in the School of Natural Sciences, Griffith University, Australia (GUTFK1-2016-1A). The kinos were thoroughly dried using a Sunbeam food dehydrator and stored at $-30^{\circ} \mathrm{C}$ until required. Prior to use, the dried kinos were ground into a coarse powder using a coffee grinder. Individual $1 \mathrm{~g}$ quantities of the powdered T. ferdinandiana kinos were weighed into separate tubes and $50 \mathrm{~mL}$ of methanol, sterile deionised water, ethyl acetate, chloroform or hexane were added. All solvents were obtained from Ajax Chemicals, Australia and were AR grade. The ground T. ferdinandiana kinos were extracted in each solvent for $24 \mathrm{~h}$ at $4^{\circ} \mathrm{C}$ with gentle shaking. The extracts were subsequently filtered through Whatman No. 54 filter paper under vacuum. The solvent extracts were air dried at room temperature. The aqueous extracts were lyophilised by freeze drying at $-50^{\circ} \mathrm{C}$. The resultant dried extracts were weighed to determine the extraction yield, and dissolved in $10 \mathrm{~mL}$ deionised water (containing 1\% DMSO) and stored at $4^{\circ} \mathrm{C}$ until use.

\section{Qualitative phytochemical studies}

Phytochemical analysis of the extracts for the presence of saponins, phenolic compounds, flavonoids, phytosterols, triterpenoids, cardiac glycosides, anthraquinones, tannins and alkaloids were conducted by standard assays. ${ }^{17-19}$

\section{Inhibitory bioactivity against Giardia duodenalis trophozoites \\ Parasite culture}

The Giardia duodenalis S-2 (sheep strain 2) trophozoite strain used in this study was a gift from Professor Andre Buret, University of Calgary, Canada. The tropozoites were maintained and subcultured anaerobically at $37^{\circ} \mathrm{C}$ in TYI-S-33 growth media supplemented with $1 \%$ bovine bile (Sigma), 10 \% Serum Supreme (Cambrex Bio products) and $200 \mathrm{IU} / \mathrm{mL}$ penicillin $/ 200 \mu \mathrm{g} / \mathrm{mL}$ streptomycin (Invitrogen, USA). Confluent mid $\log$ phase cultures were passaged every 2 days by chilling the cultures on ice for a minimum of $10 \mathrm{~min}$, followed by vortexing to dislodge the adherent trophozoites from the walls of the culture vessel. Fresh culture media $(5 \mathrm{~mL})$ was seeded with approximately $1 \times 10^{5}$ trophozoites for each passage.

\section{Evaluation of anti-Giardial activity}

Anti-Giardial activity was tested using standard assays. ${ }^{16,20,21}$ Briefly, $70 \mu \mathrm{L}$ trophozoite suspension (containing approximately $1 \times 10^{5}$ trophozoites) was dispensed into the wells of a 96 well plate. A volume of $30 \mu$ of the test extracts or the vehicle solvent or culture media (for the negative controls) was added to individual wells and the plates were incubated anaerobically at $37^{\circ} \mathrm{C}$ for $12 \mathrm{~h}$ in a humidified anaerobic atmosphere. The cell number was determined by direct enumeration and expressed as a $\%$ of the untreated control proliferation. All tests were performed in at least triplicate and triplicate controls were included on each plate.

\section{Screen for anticancer bioactivity Cancer cell lines}

The Caco2 and HeLa carcinoma cell lines used in this study were obtained from American Type Culture Collection (Rockville, USA). The cells were cultured in Roswell Park Memorial Institute (RPMI) 1640 medium (Life Technologies), supplemented with $20 \mathrm{mM}$ HEPES, $10 \mathrm{mM}$ sodium bicarbonate, $50 \mu \mathrm{g} / \mathrm{mL}$ streptomycin, $50 \mathrm{IU} / \mathrm{mL}$ penicillin, $2 \mathrm{mM}$ glutamine and $10 \%$ foetal calf serum (Life Technologies). The cells were maintained as monolayers in $75 \mathrm{~mL}$ flasks at $37^{\circ} \mathrm{C}, 5 \% \mathrm{CO}_{2}$ in a humidified atmosphere until approximately $80 \%$ confluent.

\section{Evaluation of cancer cell antiproliferative activity}

The antiproliferative activity of the extracts was assessed as previously described. ${ }^{9,10}$ Aliquots of resuspended $\mathrm{Caco} 2$ or $\mathrm{HeLa}$ cells $(70 \mu \mathrm{L}$, containing approximately 5000 cells) and $30 \mu \mathrm{L}$ of the test extracts or cell media (for the negative control) were added to individual wells of a 96 well plate and incubated at $37^{\circ} \mathrm{C}, 5 \% \mathrm{CO}_{2}$ for 12 hours in a humidified atmosphere. A volume of $20 \mu \mathrm{L}$ of Cell Titre 96 Aqueous One solution (Promega) was subsequently added to each well and the plates were incubated for a further $3 \mathrm{~h}$. Absorbances were recorded at $490 \mathrm{~nm}$ using a Molecular Devices, Spectra Max M3 plate reader. All tests were performed in at least triplicate and triplicate controls were included on each plate. The antiproliferative activity of each test was calculated as a percentage of the negative control using the following formula:

$$
\text { Proliferation }(\% \text { untreated control })=\left(\mathrm{A}_{\mathrm{ct}} / \mathrm{A}_{\mathrm{cc}}\right) \times 100
$$

$A_{c t}$ is the corrected absorbance for the test extract (calculated by subtracting the absorbance of the test extract in media without cells from the extract cell test combination) and $\mathrm{A}_{c c}$ is the corrected untreated control (calculated by subtracting the absorbance of the untreated control in media without cells from the untreated cell media combination).

\section{Toxicity screening \\ Reference toxin for toxicity screening}

Potassium dichromate $\left(\mathrm{K}_{2} \mathrm{Cr}_{2} \mathrm{O}_{7}\right)$ (AR grade, Chem-Supply, Australia) was prepared as a $1.6 \mathrm{mg} / \mathrm{mL}$ solution in distilled water and was serially diluted in artificial seawater for use in the Artemia franciscana nauplii bioassay.

\section{Artemia franciscana nauplii toxicity screening}

Toxicity was tested using a modified Artemia franciscana nauplii lethality assay as previously described. ${ }^{22,23}$ Briefly, A. franciscana nauplii were exposed to diluted plant extracts or the reference toxin and incubated at $25 \pm 1^{\circ} \mathrm{C}$ under artificial light (1000 Lux). The wells were checked after $24 \mathrm{~h}$ exposure and the $\%$ mortality per well was calculated. The $\mathrm{LC}_{50}$ with 95\% confidence limits for each treatment was calculated using probit analysis.

\section{Statistical analysis}

Data are expressed as the mean \pm SEM of at least three independent experiments. One-way ANOVA was used to calculate statistical significance between control and treated groups with a $P$ value $<0.01$ considered to be statistically significant.

\section{RESULTS}

\section{Liquid extraction yields and qualitative phytochemical} screening

Extraction of $1 \mathrm{~g}$ of the dried and powdered T. ferdinandiana kino with solvents of varying polarity yielded dried plant extracts ranging from $486 \mathrm{mg}$ (ethyl acetate extract) to $784 \mathrm{mg}$ (methanol extract) (Table 1). The dried extracts were resuspended in $10 \mathrm{~mL}$ of deionised water (containing 1\% DMSO) resulting in the extract concentrations shown in Table 1. Qualitative phytochemical studies (Table 1) determined that methanol and water extracted the widest range of phytochemicals. Both contained moderate to high levels of phenolic (both water soluble and insoluble phenolic), triterpenoids, flavonoids and tannins, as well as low 


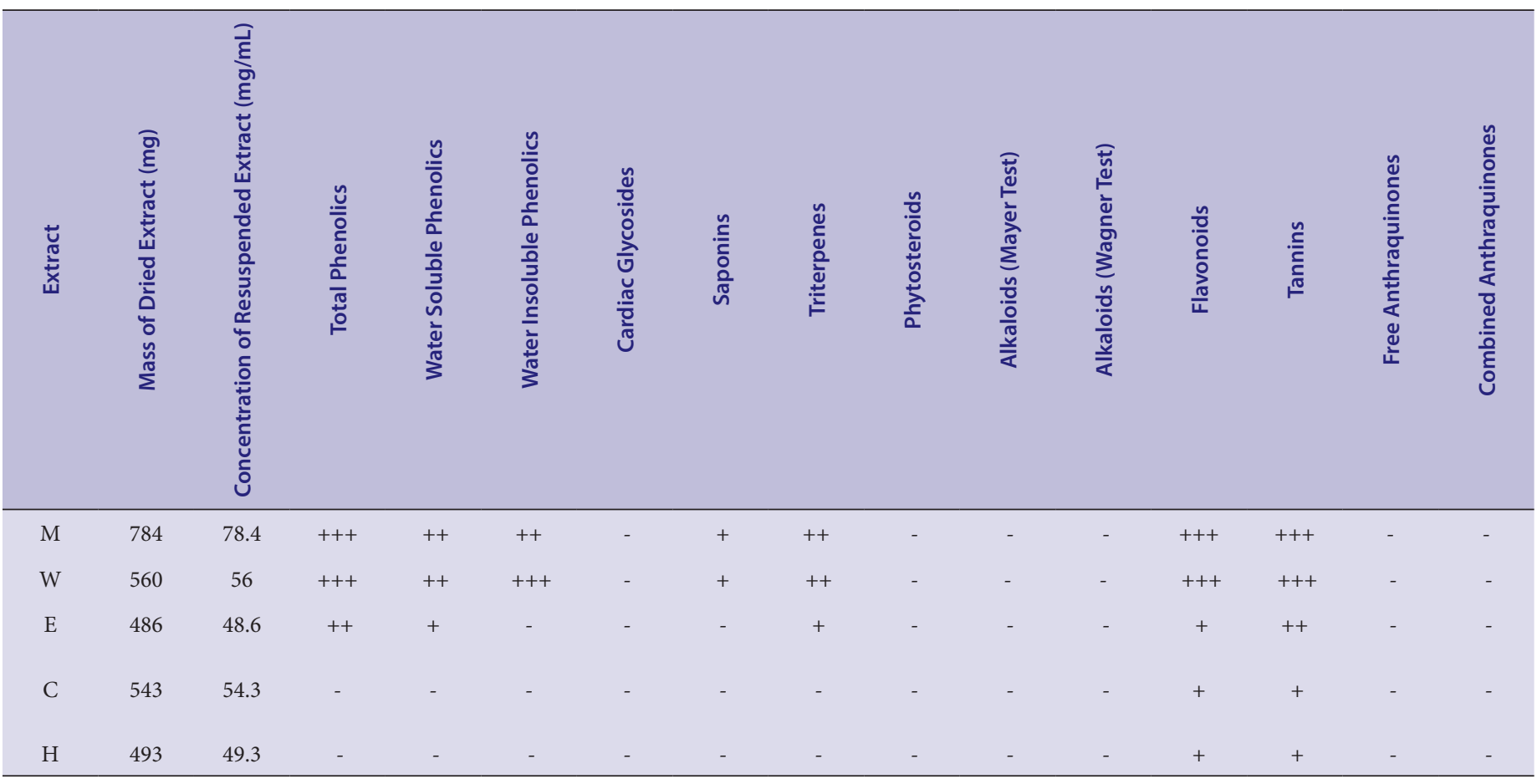

+++ indicates a large response; ++ indicates a moderate response; + indicates a minor response; - indicates no response in the assay; $\mathrm{M}=$ methanolic T. ferdinandiana kino extract; $\mathrm{W}=$ aqueous $T$. ferdinandiana kino extract; $\mathrm{E}=$ ethyl acetate $T$. ferdinandiana kino extract; $\mathrm{C}=$ chloroform $T$. ferdinandiana kino extract; $\mathrm{H}=$ hexane $T$. ferdinandiana kino extract.

levels of saponins. The ethyl acetate extract contained only moderate levels of phenolic and tannins, as well as low levels of flavonoids. Only low levels of flavonoids and tannins were detected in the chloroform and hexane extracts. Phytosterols, alkaloids, cardiac glycosides and anthraquinones were not detected in any extract.

\section{Inhibition of Giardia duodenalis proliferation}

T. ferdinandiana kino extracts were screened for their ability to inhibit G. duodenalis growth (Figure 1). The methanol, water, ethyl acetate and chloroform extracts displayed significant inhibitory activity $(\mathrm{p}<0.01)$. The methanol and water extracts were particularly potent, completely inhibiting Giardial growth. The ethyl acetate and chloroform extracts were also effective at inhibiting trophozoite growth, albeit to a lesser extent (by $\leq 25 \%$ compared to the growth of the negative controls). The hexane extract was completely ineffective against $G$. duodenalis proliferation, with no significant difference to the untreated control levels.

The T. ferdinandiana kino extracts were further tested over a range of concentrations to determine the $\mathrm{IC}_{50}$ values (Table 2) for each extract against $G$. duodenalis. Inhibition of trophozoite growth was dosedependent, with the level of inhibitory activity decreasing at lower concentrations. The methanolic and aqueous extracts were good inhibitors of G. duodenalis proliferation, with $\mathrm{IC}_{50}$ values of 996 and $1175 \mu \mathrm{g} / \mathrm{mL}$ respectively.

\section{Inhibition of cancer cell proliferation}

Aliquots of all extracts were tested for the ability to block the proliferation of Caco2 (Figure 2) and HeLa (Figure 3) cell lines. All of the T. ferdinandiana kino extracts displayed significant $(\mathrm{p}<0.01)$ antiproliferative effects against $\mathrm{Caco} 2$ cells (Figure 2). Indeed, all extracts inhibited $\mathrm{Caco} 2$ cell

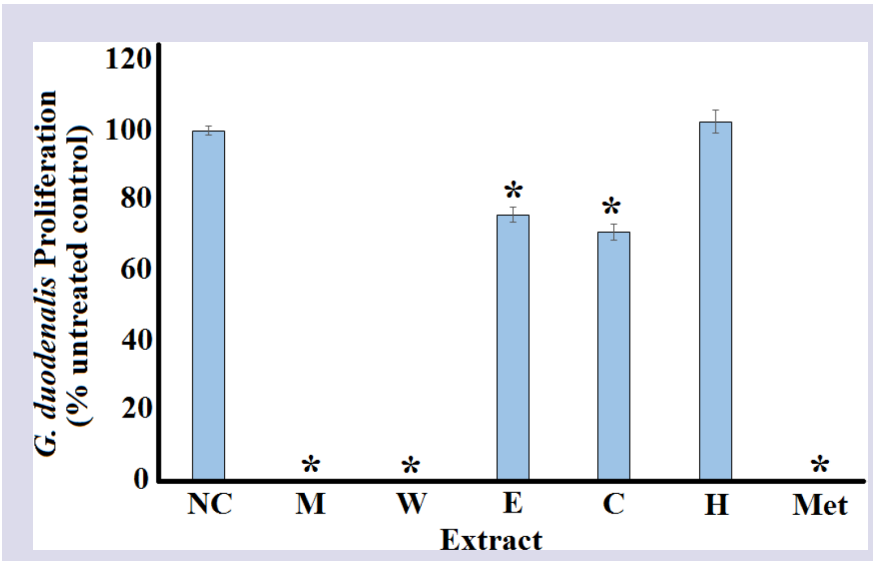

Figure 1: Inhibitory activity of the $T$. ferdinandiana kino extracts against Giardia duodenalis trophozoites measured as percentages of the untreated control cells. $\mathrm{NC}=$ untreated control; $\mathrm{M}=$ methanolic $T$. ferdinandiana extract; $\mathrm{W}=$ aqueous $T$. ferdinandiana extract; $\mathrm{E}=$ ethyl acetate $T$. ferdinandiana extract; $\mathrm{C}=$ chloroform $T$. ferdinandiana extract; $\mathrm{H}=$ hexane $T$. ferdinandiana extract; Met $=$ metronidazole control $(50 \mu \mathrm{g} / \mathrm{mL})$. Results are expressed as mean percentages \pm SEM of at least triplicate determinations. * indicates results that are significantly different to the untreated control $(p<0.01)$.

proliferation by $\geq 85 \%$, compared to the untreated control. This inhibition was particularly noteworthy and was substantially better than the cisplatin control $(50 \mu \mathrm{g} / \mathrm{mL})$, which inhibited Caco2 proliferation by $<75 \%$. The extracts were similarly effective at inhibiting HeLa cancer cell 
Table 2: The $\mathrm{IC}_{50}(\mu \mathrm{g} / \mathrm{mL})$ of the $T$. ferdinandiana kino extracts against G. duodenalis and the Caco2 and HeLa cancer cell lines, and quantification of toxicity reported as $\mathrm{LC}_{50}$ values $(\mu \mathrm{g} / \mathrm{mL})$.

\begin{tabular}{ccccc}
\hline \multirow{2}{*}{$\begin{array}{c}\text { Extract } \\
\text { G. duodenalis }\end{array}$} & \multicolumn{3}{c}{$\begin{array}{c}\text { Antiproliferative activity } \\
\left(\mathbf{I C}_{\mathbf{5 0}}: \mu \mathrm{g} / \mathrm{mL}\right)\end{array}$} & $\begin{array}{c}\text { 24 h Artemia } \\
\text { nauplii toxicity }\end{array}$ \\
\cline { 2 - 5 } & Caco2 & HeLa & LC $_{50}(\mu \mathrm{g} / \mathrm{mL})$ & \\
\hline M & 996 & 863 & 495 & 1138 \\
W & 1175 & 275 & 259 & 1370 \\
E & $\mathrm{CND}$ & 783 & 8058 & $\mathrm{CND}$ \\
$\mathbf{C}$ & $\mathrm{CND}$ & 1102 & $>10,000$ & $\mathrm{CND}$ \\
$\mathbf{H}$ & - & 1254 & $>10,000$ & $\mathrm{CND}$ \\
PC & 11 & 31 & 34 & 71 \\
\hline
\end{tabular}

Numbers indicate the mean $\mathrm{IC}_{50}$ or $\mathrm{LC}_{50}$ values of triplicate determinations. - indicates that inhibition was not evident at any dose tested. CND indicates that $\mathrm{IC}_{50}$ or $\mathrm{LC}_{50}$ values could not be obtained as the $\%$ inhibition or $\%$ mortality did not exceed $50 \%$ at any dose tested.

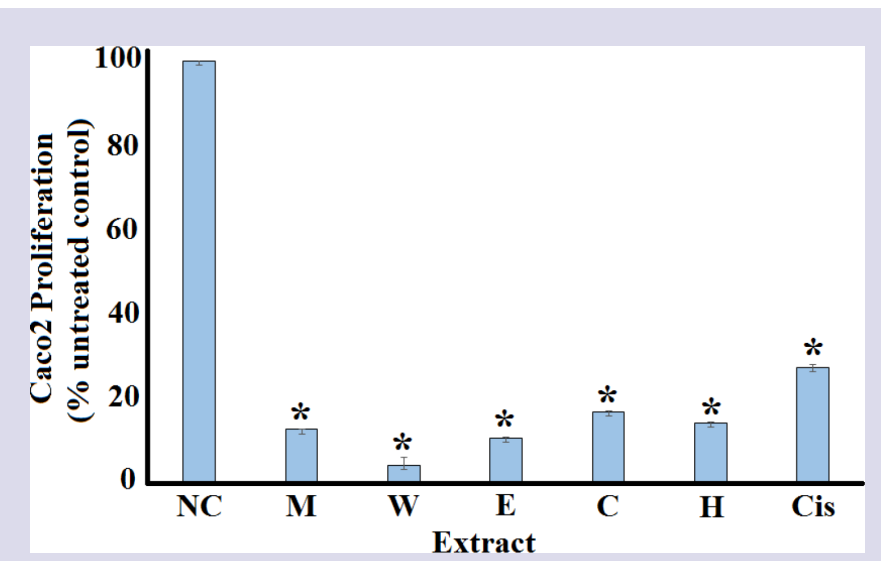

Figure 2: Anti-proliferative activity of the T. ferdinandiana kino extracts and untreated controls against $\mathrm{Caco} 2$ cancer cell lines measured as percentages of the untreated control cells. NC = untreated control; $\mathrm{M}=$ methanolic $T$. ferdinandiana extract; $\mathrm{W}=$ aqueous $T$. ferdinandiana extract; $\mathrm{E}=$ ethyl acetate $T$. ferdinandiana extract; $\mathrm{C}=$ chloroform $T$. ferdinandiana extract; $\mathrm{H}$ = hexane $T$. ferdinandiana extract; Cis $=$ cisplatin control $(50 \mu \mathrm{g} / \mathrm{mL})$. Results are expressed as mean percentages \pm SEM of at least triplicate determinations. * indicates results that are significantly different to the untreated control $(p<0.01)$.

proliferation (Figure 3), although the mid to lower polarity ethyl acetate, chloroform and hexane extracts were less potent inhibitors of HeLa cell proliferation compared to Caco 2 proliferation (judged by the \% proliferation inhibition).

The antiproliferative efficacy of the T. ferdinandiana kino extracts was further quantified by determining the $\mathrm{IC}_{50}$ values for each extract which inhibited cell proliferation against each cell line (Table 2). The aqueous and methanolic extracts were particularly effective at inhibiting cancer cell proliferation, with $\mathrm{IC}_{50}$ values substantially $<1000 \mu \mathrm{g} / \mathrm{mL}$ against both $\mathrm{Caco} 2$ and $\mathrm{HeLa}$ cell lines. The aqueous extract was the most potent inhibitor of Caco2 and HeLa proliferation, with $\mathrm{IC}_{50}$ values of 275 and $259 \mu \mathrm{g} / \mathrm{mL}$ respectively.

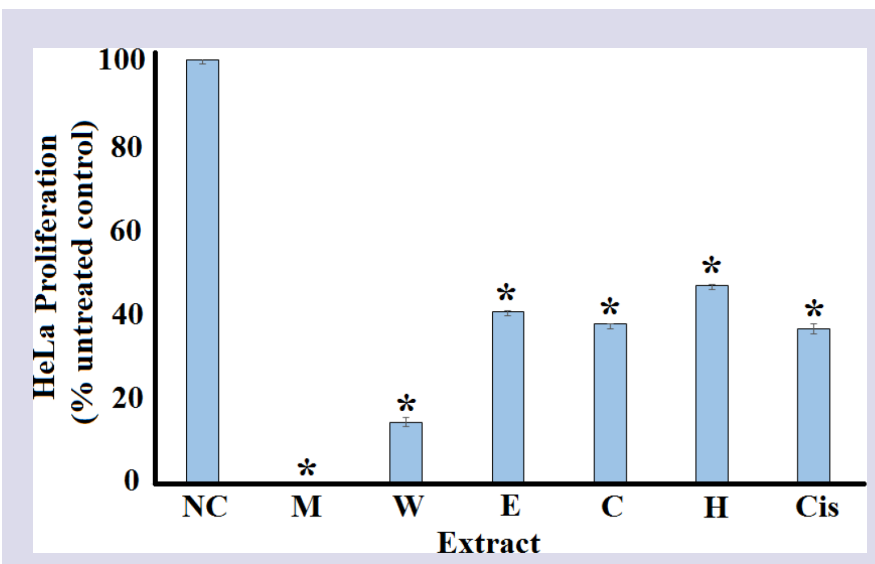

Figure 3: Anti-proliferative activity of the T. ferdinandiana kino extracts and untreated controls against HeLa cancer cell lines measured as percentages of the untreated control cells. NC = untreated control; $\mathrm{M}=$ methanolic $T$. ferdinandiana extract; $\mathrm{W}=$ aqueous $T$. ferdinandiana extract; $\mathrm{E}=$ ethyl acetate $T$. ferdinandiana extract; $\mathrm{C}=$ chloroform $T$. ferdinandiana extract; $\mathrm{H}=$ hexane $T$. ferdinandiana extract; Cis = cisplatin control $(50 \mu \mathrm{g} / \mathrm{mL})$. Results are expressed as mean percentages \pm SEM of at least triplicate determinations. * indicates results that are significantly different to the untreated control $(p<0.01)$.

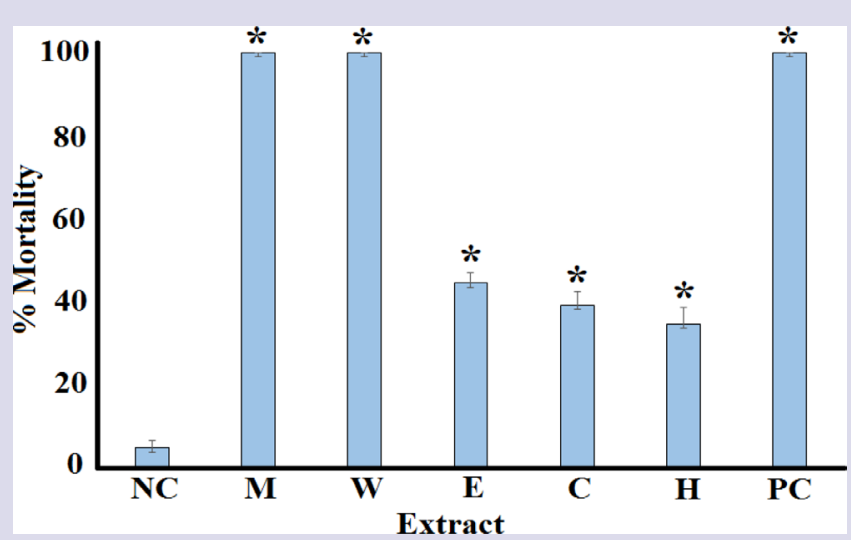

Figure 4: The lethality of the T. ferdinandiana kino extracts and the potassium dichromate control $(1000 \mu \mathrm{g} / \mathrm{mL})$ towards Artemia nauplii following 24 hours exposure. $\mathrm{NC}=$ untreated control; $\mathrm{M}=$ methanolic T. ferdinandiana extract; $\mathrm{W}=$ aqueous $T$. ferdinandiana extract; $\mathrm{E}=$ ethyl acetate $T$. ferdinandiana extract; $C=$ chloroform $T$. ferdinandiana extract; $\mathrm{H}=$ hexane $T$. ferdinandiana extract; $\mathrm{PC}=$ positive control $(1000 \mu \mathrm{g} / \mathrm{ml}$ potassium dichromate). All tests were performed in at least triplicate and the results are expressed as mean \pm SEM. ${ }^{*}$ indicates results that are significantly different to the negative control $(p<0.01)$.

\section{Quantification of toxicity}

All extracts were initially screened at $2000 \mu \mathrm{g} / \mathrm{mL}$ in the Artemia nauplii bioassay following $24 \mathrm{~h}$ exposure (Figure 4). For comparison, the reference toxin potassium dichromate $(1000 \mu \mathrm{g} / \mathrm{mL})$ was also tested. The potassium dichromate reference toxin was rapid in its onset of mortality, inducing mortality within the first $3 \mathrm{~h}$ of exposure and $100 \%$ mortality was evident following 4-5 h (unpublished results). Similarly, the methanolic and aqueous T. ferdinandiana kino extracts displayed $100 \%$ mortality rates at $24 \mathrm{~h}$ and were thus deemed to be toxic. All other extracts induced levels of mortality which were below $50 \%$ and were thus deemed to be 
nontoxic.

To further quantify the effect of toxin concentration on the induction of mortality, the extracts were serially diluted in artificial seawater to test across a range of concentrations in the Artemia nauplii bioassay. Table 2 shows the $\mathrm{LC}_{50}$ values of the extracts towards $A$. franciscana. No $\mathrm{LC}_{50}$ values are reported for the ethyl acetate, chloroform or hexane T. ferdinandiana kino extracts as less than $50 \%$ mortality was seen for all concentrations tested. Extracts with $\mathrm{LC}_{50}$ values $>1000 \mu \mathrm{g} / \mathrm{mL}$ towards Artemia nauplii have been defined as being nontoxic. ${ }^{24}$ As $\mathrm{LC}_{50}$ values $>1000 \mu \mathrm{g} / \mathrm{mL}$ were determined for the methanolic and aqueous extracts, they were also deemed to be nontoxic.

\section{DISCUSSION}

T. ferdinandiana fruit is a highly nutritious food and the leaves and kinos have been used as traditional Australian Aboriginal medicines for thousands of years. ${ }^{12}$ Due to its extremely high antioxidant content, ${ }^{25}$ most studies into the therapeutic properties of T. ferdinandiana have focused on fruit extracts. Several studies have reported potent antibacterial properties for fruit solvent extracts against food spoilage ${ }^{19}$ and food poisoning bacteria, ${ }^{19,26}$ as well against the bacterial triggers of rheumatoid arthritis ${ }^{14}$ and multiple sclerosis. ${ }^{15}$ It has also been reported to be a potent growth inhibitor of the endospore forming pathogens Bacillus anthracis ${ }^{27}$ and Clostridium perfringens. ${ }^{28}$ Several studies have also tested extracts prepared from T. ferdinandiana leaves on similar bacterial species and report higher potency compared to the equivalent fruit extracts. ${ }^{13,27,28,29}$ Extracts produced from the fruit are also potent inhibitors of the proliferation of the gastrointestinal protozoal parasite, Giardia duodenalis. ${ }^{16}$ However, we were unable to find any studies that tested leaf or kino extracts against G. duodenalis, or other protozoal parasites. Several studies have also reported that $T$. ferdinandiana fruit extracts have anti-proliferative activity against several cancer cell lines. ${ }^{7,8}$ These studies reported that the T. ferdinandiana fruit extracts blocked proliferation via apoptosis induction. ${ }^{8}$ Interestingly, one of these studies also tested leaf extracts and reported similar activity but stronger potency compared to the fruit extract. ${ }^{7}$ Despite the previous studies examining the therapeutic properties of other parts of the plant and their use in traditional medicine, T. ferdinandiana kinos remain to be evaluated for their therapeutic properties.

The current study examined the anti-proliferative properties of T. ferdinandiana kino extracts against the food/water borne gastrointestinal parasite G. duodenalis, and against two cancer cell lines. Potent growth inhibition of $G$. duodenalis was noted for the kino extracts. Giardial infection (giardiasis) is a re-emerging disease which afflicts large numbers of individuals worldwide, with higher incidence in countries with poorer socio-economic conditions, inadequate sanitary conditions, untreated water supplies and poor diet. ${ }^{30}$ Whilst generally not fatal, giardiasis results in debilitating symptoms including bloating, diarrhoea, excess gas, loss of appetite, loose and watery stool, stomach cramps and haematuria. Currently, there are only a narrow range of drugs effective against giardiasis, including quinalones and imidazole derivatives. None of these drugs is ideal as they produce unpleasant side effects including nausea, vertigo, vomiting, diarrhoea and hallucinations. ${ }^{30,31}$ Furthermore, recent reports of the failure of current treatments to address this disease indicate a developing drug resistance of several Giardia strains. ${ }^{31}$ Recent studies have highlighted the potential of plant medicines and have demonstrated that some plant components are very effective inhibitors of Giardia duodenalis growth, with similar potency to the gold standard drug metronidazole ${ }^{16,20}$ Our studies demonstrate that T. ferdinandiana kino extracts also possess anti-Giardial activity. Whilst, further studies are required to identify the active component(s), previous studies have identified several tannins and lactone compounds in extracts produced from the leaves of this species. Notably, all these compounds have inhibitory activity against amoebic protozoans. ${ }^{16}$ It is possible that these compounds may also contribute to the anti-Giardial activity seen in our study, although further tests are required to determine if they are present in the kino extracts.

The antiproliferative efficacy of the T. ferdinandiana kino extracts was also examined against two human cancer cell lines in vitro; $\mathrm{Caco} 2$ (colorectal) and HeLa (cervical). Anti-proliferative activity was evident for all the T. ferdinandiana kino extracts, although it was particularly noteworthy for the methanol and water and extracts, with $\mathrm{IC}_{50}$ values substantially $<1000 \mu \mathrm{g} / \mathrm{mL}$. The aqueous extract was a particularly potent inhibitor of Caco2 2 and HeLa proliferation, with $\mathrm{IC}_{50}$ values of approximately 275 and $260 \mu \mathrm{g} / \mathrm{mL}$ respectively. These findings support and extend previous studies examining the anticancer effects of T. ferdinandiana fruit ${ }^{7,8}$ and leaf extracts ${ }^{7}$ against these and other cell lines. The earlier studies also examined the antiproliferative mechanism and determined that the extracts induced apoptosis. ${ }^{78}$ Furthermore, as the fruit extracts activate caspase-7, caspase- 9 and poly (ADP-ribose) polymerase (PARP), it is likely that apoptosis induction is via an intrinsic pathway. ${ }^{8}$ Our study did not examine the antiproliferative mechanism of the T. ferdinandiana kino extracts. Nor did we identify the active components of the cytotoxic extracts. Further isolation and identification studies are required to determine these details.

The findings reported here also demonstrate that all $T$. ferdinandiana kino extracts were nontoxic towards $A$. franciscana nauplii. Extracts with LC $_{50}$ values $>1000 \mu \mathrm{g} / \mathrm{mL}$ towards Artemia nauplii have been defined as being nontoxic. ${ }^{24}$ However, further studies using normal human cell lines are required to verify the safety of these extracts for therapeutic use. Furthermore, bioactivity driven separation studies are required to isolate the active components and determine their mechanism of action. The results of this study indicate that the $T$. ferdinandiana kino extracts examined in this report are worthy of further study due to their antiGiardial activity and ability to block cancer Caco2 and HeLa carcinoma cell proliferation. Further evaluation of the anti-Giardial and anticancer properties of these extracts is warranted. Likewise, bioactivity driven purification studies are needed to examine the mechanisms of action of these agents.

\section{CONCLUSION}

The potent antiproliferative activity and lack of toxicity of the methanolic and aqueous T. ferdinandiana kino extracts indicates their potential in the treatment and prevention of giardiasis and some cancers. Purification and identification of the bioactive components is needed to examine the mechanisms of action of these agents.

\section{ACKNOWLEDGEMENT}

The authors are grateful to David Boehme of Wild Harvest, Northern Territory, Australia for providing the T. ferdinandiana kinos used in these studies. We are also thankful to Professor Andre Buret, University of Calgary, Canada for the gift of the G. duodenalis S-2 (sheep strain 2) trophozoite strain used in this. Financial support for this work was provided by the Environmental Futures Research Institute, Griffith University.

\section{REFERENCES}

1. Cock IE. The medicinal properties and phytochemistry of plants of the genus Terminalia (Combretaceae). Inflammopharmacology 2015;23(5):203-29. DOI 10.1007/s10787-015-0246-z.

2. Sandhya T, Lathika KM, Pandey BN, et al. Potential of traditional Ayurvedic formulation, triphala, as a novel anticancer drug. Cancer Letters. 2006;231(2):206-14.

3. Kaur S, Michael H, Arora S, et al. The in vitro cytotoxic and apoptotic activity of Triphala: An Indian herbal drug. Journal of Ethnopharmacology. 2005;97(1):15-20. 
4. Shi Y, Sahu RP, Srivastava SK. Triphala inhibits both in vitro and in vivo xenograft growth of pancreatic tumor cells by inducing apoptosis. BMC Cancer. 2008;8(1):294.

5. Sandhya T, Mishra KP. Cytotoxic response of breast cancer cell lines, MCF-7 and $T 47 \mathrm{D}$ to triphala and its modification by antioxidants. Cancer Letters. 2006;238(2):304-13

6. Pinmai K, Chunlaratthanabhorn S, Ngamkitidechakul C, et al. Synergistic growth inhibitory effects of Phyllanthus emblica and Terminalia bellerica extracts with conventional cytotoxic agents: Doxorubicin and cisplatin against human hepatocellular carcinoma and lung cancer cells. World Journal of Gastroenterology. 2008;14(10):1491-7.

7. Shalom J, Cock IE. Terminalia ferdinandiana Exell. Fruit and leaf extracts inhibit proliferation and induce apoptosis in selected human cancer cell lines. Nutrition and Cancer; in press.

8. Tan AC, Konczak I, Ramzan I, et al. Potential antioxidant, antiinflammatory, and proapoptotic anticancer activities of Kakadu plum and Illawarra plum polyphenolic fractions. Nutrition and Cancer. 2011;63(7):1074-84.

9. Courtney R, Sirdaarta J, White A, et al. Inhibition of Caco-2 and HeLa proliferation by Terminalia carpentariae C.T.White and Terminalia grandiflora Benth. Extracts: Identification of triterpenoid components. Pharmacognosy Journal. 2017;9(4):175-84. DOI: 10.5530/pj.2017.2.29.

10. Jamieson N, Sirdaarta J, Cock IE. The anti-proliferative properties of Australian plants with high antioxidant capacities against cancer cell lines. Pharmacognosy Communications 2014;4(4):71-82. DOI: 10.5530/pc.2014.4.8.

11. Eberhardt MV, Lee CY, Liu RH. Nutrition: Antioxidant activity of fresh apples. Nature. 2000;405(6789):903-4

12. Cock IE. Medicinal and aromatic plants - Australia, in Ethnopharmacology section, Biological, Physiological and Health Sciences, Encyclopedia of Life Support Systems (EOLSS). 2011. Developed under the Auspices of the UNESCO, EOLSS Publishers, Oxford ,UK, (http://www.eolss.net)

13. Courtney R, Sirdaarta J, Matthews B, et al. Tannin components and inhibitory activity of Kakadu plum leaf extracts against microbial triggers of autoimmune inflammatory diseases. Pharmacognosy Journal. 2015;7(1):18-31. DOI: 10.5530/ pj.2015.7.2.

14. Sirdaarta J, Matthews B, Cock IE. Inhibitory activity of Kakadu plum fruit extracts against microbial triggers of rheumatoid arthritis: Identification of stilbene and tannin components. Journal of Functional Foods. 2015;17(1):610-20. http:// dx.doi.org/10.1016/j.jff.2015.06.019.

15. Sirdaarta J, Matthews B, White A, et al. GC-MS and LC-MS analysis of Kakadu plum fruit extracts displaying inhibitory activity against microbial triggers of multiple sclerosis. Pharmacognosy Communications. 2015;5(2):100-15. DOI: 10.5530/pc.2015.2.2

16. Rayan $\mathrm{P}$, Matthews $B, M c D o n n e l l ~ P$, et al. Terminalia ferdinandiana extracts as inhibitors of Giardia duodenalis proliferation: A new treatment for giardiasis. Parasitology Research. 2015;114(7):2611-20. DOI 10.1007/s00436-015-4465-4.

17. Lee CJ, Wright MH, Arnold MSJ, et al. Inhibition of Streptococcus pyogenes growth by native Australian plants: New approaches towards the management of impetigo, pharyngitis and rheumatic heart disease. Pharmacognosy Communications. 2016;6(3):164-73. DOI: 10.5530/pc.2016.3.6.

18. Hart C, Illanko P, Sirdaarta J, et al. Tasmannia stipitata as a functional food/ natural preservative: Antimicrobial activity and toxicity. Pharmacognosy Communications. 2014;4(4):33-47. DOI: 10.5530/pc.2014.4.4.

19. Mohanty S, Cock IE. The chemotherapeutic potential of Terminalia ferdinandiana: Phytochemistry and bioactivity. Pharmacognosy Reviews. 2012;6(11):29-36. DOI: 10.4103/0973-7847.95855.

20. Rayan $\mathrm{P}$, Matthews $\mathrm{B}, \mathrm{McD}$ onnell $\mathrm{P}$, et al. Phytochemical analysis of Tasmannia lanceolata extracts and inhibition of Giardia duodenalis proliferation. Pharmacognosy Journal. 2016;8(3):291-9. DOI: 10.5530/pj.2016.3.19

21. Arkhipov A, Sirdaarta J, Rayan $P$, et al. An examination of the antibacterial, antifungal, anti-Giardial and anticancer properties of Kigelia africana fruit extracts. Pharmacognosy Communications 2014;4(3):62-76. DOI: 10.5530/pc.2014.3.7.

22. Wright MH, Matthews $B$, Arnold MSJ, et al. The prevention of fish spoilage by high antioxidant Australian culinary plants: Shewanella putrafaciens growth inhibition. International Journal of Food Science and Technology. 2016;51(3):801-13. doi:10.1111/ijfs. 13026

23. Mpala L, Chikowe G, Cock IE. No evidence of antiseptic properties and low toxicity of selected Aloe species. Journal of Pharmaceutical Negative Results. 2010;1(1):10-6. DOI: 10.4103/0976-9234.68869.

24. Cock IE, Ruebhart DR. Comparison of the brine shrimp nauplii bioassay and the ToxScreen-II test for the detection of toxicity associated with Aloe vera (Aloe barbadensis Miller) leaf extract. Pharmacognosy Research. 2009;1(2):98-101.

25. Netzel M, Netzel G, Tian Q, et al. Native Australian fruits - a novel source of antioxidants for food. Innovative Food Science and Emerging Technologies. 2007:8(3):339-46.

26. Wright $\mathrm{MH}$, Arnold MS, Aldosary $\mathrm{H}$, et al. Bioactive constituents of Terminalia ferdinandiana Exell: A pharmacognostic approach towards the prevention and treatment of yersiniosis. Pharmacognosy Communications. 2016;6(3):152-63. DOI: $10.5530 / p c .2016 .3 .5$

27. Wright $\mathrm{MH}$, Sirdaarta J, White A, et al. GC-MS headspace analysis of Terminalia ferdinandiana fruit and leaf extracts which inhibit Bacillus anthracis growth. Pharmacognosy Communications. 2017;9(1):73-82. DOI: 10.5530/pj.2017.1.14.

28. Wright MH, Sirdaarta J, Matthews B, et al. Growth inhibitory activity of Kakadu plum extracts against the opportunistic pathogen Clostridium perfringens: New leads in the prevention and treatment of clostridial myonecrosis. Pharmacognosy Journal. 2016;8(2):144-53. DOI: 10.5530/pj.2016.2.8.

29. McManus $\mathrm{K}$, Wood A, Wright $\mathrm{MH}$, et al. Terminalia ferdinandiana Exell. Extracts inhibit the growth of body odour-forming bacteria. International Journal of Cosmetic Science. 2017;39(5):500-10. DOI: 10.1111/ics.12403.

30. Rayan P, Stenzel D, McDonnell PA. The effects of saturated fatty acids on Giardia duodenalis trophozoites in vitro. Parasitology Research. 2005;97(3):191-200.

31. Harris JC, Plummer S, Lloyd D. Anti-giardial drugs. Applied Microbiology and Biotechnology. 2001;57(5-6):614-9.
PICTORIAL ABSTRACT
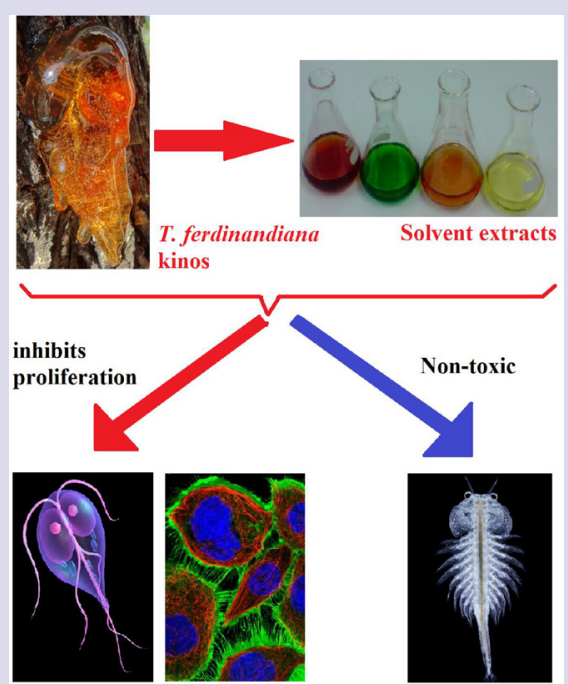

G. duodenalis
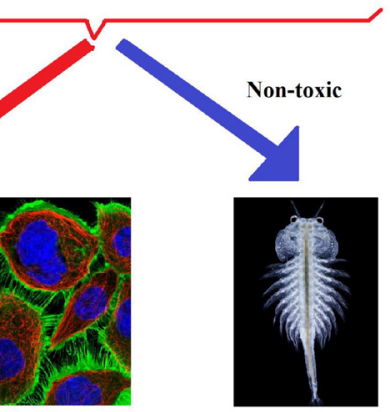

A. franciscane nauplii

\section{HIGHLIGHTS OF PAPER}

- Methanolic and aqueous T. ferdinandiana kino extracts were strong inhibitors of Caco2 and HeLa cell proliferation.

- The aqueous extract was particularly potent against Caco2 and HeLa cells $\left(\mathrm{IC}_{50} \sim\right.$ 275 and $260 \mu \mathrm{g} / \mathrm{mL}$ respectively).

- The mid to lower polarity solvent extractions were less potent inhibitors of cell proliferation.

- The aqueous and methanolic extracts were also effective inhibitors of $G$. duodenalis proliferation (IC $\mathrm{C}_{50}$ values of 1000 and $1200 \mu \mathrm{g} / \mathrm{mL}$ respectively).

- All extracts were non-toxic in the Artemia nauplii assay.

\section{ABOUT AUTHORS}

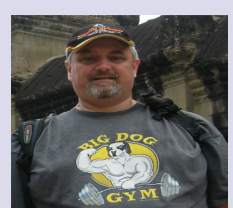

Dr. I. E. Cock leads a research team in the Environmental Futures Research Institute and the School of Natural Sciences at Griffith University, Australia. His research involves bioactivity and phytochemical studies into a variety of plant species of both Australian and international origin, including Aloe vera, South Asian and South American tropical fruits, as well as Australia plants including Scaevola spinescens, Pittosporum phylliraeoides, Terminalia ferdinandiana (Kakadu plum), Australian Acacias, Syzygiums, Petalostigmas and Xanthorrhoea johnsonii (grass trees). This range of projects has resulted in nearly 200 publications in a variety of peer reviewed journals. 